\title{
The validation of the Peer Assessment Rating index for malocclusion severity and treatment difficulty
}

\author{
L. DeGuzman, DMD, ${ }^{a}$ D. Bahiraei, BS, DMD, ${ }^{a}$ K. W. L. Vig, BDS, MS, FDS, D.Orth., ${ }^{b}$ \\ P. S. Vig, BDS, FDS, D.Orth., PhD, ${ }^{\circ}$ R. J. Weyant, MS, DMD, PhD, ${ }^{d}$ and \\ K. O'Brien, FDS, D.Orth., MSc, PhD \\ Manchester, England, and Pittsburgh, Pa.
}

The Peer Assessment Rating (PAR) index is a British occlusal index that measures the severity of dental malocclusion and has been used in several investigations that have evaluated the effectiveness of orthodontic treatment provision in Europe. As part of its development, the PAR index was validated for malocclusion severity, by using the opinions of a panel of 74 dentists and orthodontists. The present investigation was carried out to validate the PAR index, by using the opinion of an American panel of orthodontists. Eleven orthodontists examined a sample of 200 sets of study casts and rated them for malocclusion severity and perceived treatment difficulty. Multiple regression techniques were used to evaluate the predictive power of the components of malocclusion on the panel's scores. Weightings were calculated from the partial regression coefficients and, when these weightings were applied to the PAR index, the association between the panel's opinion and the PAR index scores was increased. (AM J ORTHOD DENTOFAC ORTHOP $1995 ; 107: 172-6$.

$I_{t}$ is evident to orthodontists that alternative types of orthodontic therapy achieve some measure of success, but no one treatment predictably achieves all treatment objectives all of the time. The selection from possible alternatives treatments should ideally be based on known estimates of efficacy, rather than be dependent on anecdotal clinical impression. However, to obtain data on treatment efficacy, it is necessary to use both valid and reliable measures of outcome. In this context, validity means that a specific measure actually represents the attribute that it is attempting to measure. In other words, measures of outcome must directly and accurately correspond with the degree of severity of the condition over the entire spectrum of variability.

The development of appropriate outcome measures for many medical and dental specialties is difficult to achieve because the results of treatment are frequently not well documented. Fortunately, the orthodontic specialty is unique in recording the results of treatment by study casts, photographs and radiographs, and measures that evaluate im-

From the University of Pittsburgh, School of Dental Medicine.

Supported by NIH/NIDR grant DE 09883 .

${ }^{a}$ Graduate student, Department of Orthodontics.

${ }^{b}$ Professor and Chairman, Department of Orthodontics.

'Professor and Associate Dean for Research, Dental Medicine Research Office.

${ }^{\mathrm{d} A s s i s t a n t}$ Professor, Department of Public Health and Community Dentistry.

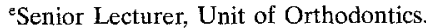

Copyright $(1) 1995$ by the American Association of Orthodontists. $0889-5406 / 95 / \$ 3.00+0 \quad \mathbf{8 / 1 / 4 9 2 9 9}$ portant elements of the outcomes of treatment, in terms of morphologic change, can be applied readily to these records.

\section{OCCLUSAL INDICES AS MEASURES OF MALOCCLUSION SEVERITY}

A practical and relatively simple measure that might be used for orthodontic outcomes research is an occlusal index. It has been suggested that the "ideal" occlusal index should possess the following properties: ${ }^{1-3}$

1. Reliability. The index shold be able to measure consistently at different times and when used by different examiners.

2. Validity. The index should measure what it purports to measure.

3. It should be amenable to modification.

4. It must yield quantitative data.

5. It should lend itself to the rapid application by trained examiners.

One occlusal index that satisfies these criteria is the Peer Assessment Rating (PAR) index. ${ }^{3}$ This is a measure of occlusal change that allocates scores to (1) alignment of the dentition (including impactions), (2) buccal segment relationship, (3) overjet, (4) overbite, and (5) midline discrepancy. It is applied to pretreatment and posttreatment dental casts and the change in PAR scores thus reflects the treatment effect on the dental occlusion and alignment.

In developing this index, a validation study was 
carried out that involved 74 British orthodontists. Their opinion on dental malocclusion severity and success of treatment was recorded for a sample of 272 dental casts. Weightings were calculated and applied to the component of PAR, and this resulted in the PAR index scores reflecting contemporary British orthodontic opinion. Arguably, a limitation of the PAR index may reside in that the British opinion may not reffect the views of orthodontists in other countries. The purpose of this study was therefore to validate the PAR index by a group of orthodontists in the United States.

\section{THE ASSESSMENT OF TREATMENT DIFFICULTY}

Several investigators have been conducted to evaluate the effectiveness of orthodontic treatment. ${ }^{4-6}$ Although these studies have provided useful data, they invariably ignored the aspects of treatment difficulty. This is an important variable, as it may affect treatment goals and in some instances may influence clinicians to accept an attainable but less than ideal treatment result. Despite the clear need for an objective measure, scant attention has been devoted to this issue. A recent study by Rowe ${ }^{7}$ reported the results of 30 orthodontists examining the pretreatment records for six cases and giving their opinion regarding difficulty of treatment. The study casts were then scored with an objective measure of malocclusion, the Summers' Occlusal Index. ${ }^{2}$ It was concluded that treatment difficulty and malocclusion severity were distinct but related entities, although various components of malocclusion were not reliable predictors of perceived treatment difficulty.

The purpose of this study was to (1) evaluate the relationship beween the subjective estimates of severity of malocclusion and treatment difficulty by using a panel of American orthodontists; (2) evaluate the relationship between severity and difficulty, and the PAR index; and (3) calculate weightings for the components of PAR to enable adaptation of the PAR index to reflect both the perceived malocclusion severity and the treatment difficulty.

\section{MATERIALS AND METHODS}

\section{The selection criteria}

We calculated the sample size for this investigation by using the data from a previous study. ${ }^{3}$ To achieve a study with a power of 0.80 at an alpha level of 0.05 , the required number of examiners was calculated to be 11 and the number of study casts as 200 .

\section{The sample of study casts}

Two hundred sets of study casts representing all malocclusion types were selected from the records of the orthodontic department at University of Pittsburgh School of Dental Medicine. Each set of study casts were scored by two examiners who were previously calibrated in the use of the PAR index. The mean of the examiners scores for the PAR components was calculated for each cast.

\section{The collection of the opinions of the orthodontists}

A group of 11 volunteer orthodontists in private practice in western Pennsylvania was convened, and each rater examined the 200 study casts. For each cast they gave their opinion regarding:

1. The degree of deviation from ideal occlusion.

2. The anticipated difficulty of treatment.

3. Their perception of the approximate duration of treatment in months.

The degree of deviation was recorded on a 5-point visual analogue scale anchored with the terms "no deviation" $=1$ to "very great deviation" $=5$. Treatment difficulty was recorded on a similar scale that was anchored with the terms "very easy" to "very difficult." To check that the raters were consistent in their opinion, they all repeated their assessment 4 weeks later on a randomly selected sample of 50 sets of casts drawn from the total sample of 200 .

\section{Data analysis}

The data were analyzed according to the following steps.

1. The consistency of the panel's opinion scores between first and second session was tested with the intraclass correlation coefficient and bias was evaluated with a one-way analysis of variance.

2. Pearson's correlation coefficient was used to evaluate the associations between the subjective opinion of the raters regarding malocclusion severity, treatment difficulty, and unweighted PAR index scores.

3. Separate multiple linear regression models were constructed to assess the association between the components of the PAR index and perceived malocclusion severity and treatment duration.

4. The resultant partial regression coefficients were then multiplied by an appropriate constant and rounded to the nearest whole number to provide weightings for the individual components of the index.

5. The weightings were applied to the components of the index and the degree of association between the weighted PAR scores and the subjective opinion of the raters was assessed with Pearson's correlation.

6. The differences between the PAR scores that corresponded to the subjective assessments of severity and difficulty were evaluated with analysis of variance and a Tukey multiple comparison post hoc test. 
Table I. The Pearsons' correlation coefficients between the panel's perception of malocclusion severity, treatment difficulty, duration of treatment, and unweighted PAR scores

\begin{tabular}{l|c|c|c}
\hline & Perceived difficulty & Duration of treatment & PAR score \\
\hline Perceived severity & 0.93 & 0.9 & 0.70 \\
Perceived difficulty & & 0.9 & 0.59 \\
Duration of treatment & & & 0.57 \\
\hline
\end{tabular}

Table II. The final regression analysis with malocclusion severity as the dependent variable

\begin{tabular}{l|c|c|c|c}
\hline \multicolumn{1}{c|}{ Term } & Beta & Sum of squares & F Ratio & Prab $>$ F \\
\hline Overjet & 0.24 & 16.06 & 85.00 & 0.000 \\
Overbite & 0.16 & 4.07 & 21.54 & 0.000 \\
Midline & 0.15 & 1.22 & 6.49 & 0.011 \\
Buccal occlusion & 0.12 & 14.46 & 76.56 & 0.000 \\
Upper anterior crowding & 0.05 & 5.68 & 30.10 & 0.000 \\
R square & 0.69 & & & \\
\hline
\end{tabular}

Equation: Severity of malocclusion $=1.67+0.24$ (overjet) +0.16 (overbite) +0.15 (midline) +0.12 (buccal occlusion) +0.05 (upper anterior crowding).

\section{RESULTS}

\section{Characteristics of the panel of raters}

The panel was comprised of eight male and three female orthodontists. Their date of dental school graduation ranged from 1952 to 1987 . The completion of their orthodontic specialty training ranged from 1956 to 1990 . Six of the participants had graduated from the University of Pittsburgh orthodontic program. All routinely provided care for all types of orthodontic patients. Nine practiced with edgewise techniques, the remaining two treated equally frequently with the Begg and Edgewise techniques. Their mean number of hours spent in providing patient care per week was 39 hours with a range of 28 to 50 hours. The number of patients that each practitioner had treated in the last year ranged from 75 to 1000 with a mean of 295.

\section{Raters reliability in the perception of malocclusion severity and treatment difficulty}

High intrarater agreement was found between the first and second sessions $(r=0.98, p<0.01)$. No evidence of bias between sessions was detected.

\section{The results of the correlation and regression analyses}

Table I reports the correlation matrix of the association between perceived malocclusion severity, treatment difficulty, duration of treatment, and unweighted PAR scores. The close association be- tween malocclusion severity and treatment difficulty may be interpreted by the suggestion that the panel's scores for severity, may have influenced the scores for difficulty. Therefore we selected the perceived duration of treatment as an estimate of treatment difficulty. The result of both initial regression analyses, with malocclusion severity and perceived treatment duration as dependent variables, revealed that buccal segment alignment and lower anterior crowding were not predictors of either dependent variable. These variables were eliminated and the resultant models for malocclusion severity and treatment difficulty are shown in Tables II and III, respectively. The weighting derived from the partial regression coefficient for the individual components of PAR for malocclusion severity and duration of treatment are shown in Table IV. When these weightings were applied to the PAR scores the correlation between PAR scores and panel's rating for malocclusion severity was increased to $r=0.83$ and for treatment difficulty it was raised to $r=0.68$.

The two sets of weightings for malocclusion severity and treatment difficulty were very similar. To simplify the use of the validated index, we combined the two sets of weightings, so that a single set of scores would represent both perceived malocclusions severity and treatment difficulty. These weights are also included in Table IV. The application of these weights resulted in a slight reduction in the Pearson's correlation between per- 
Table III. The final regression analysis with duration of treatment as the dependent variable

\begin{tabular}{|c|c|c|c|c|}
\hline Term & Beta & Sum of squares & F Ratio & Prob $>F$ \\
\hline Overjet & 0.96 & 234.94 & 85.00 & 0.000 \\
\hline Overbite & 0.75 & 75.76 & 21.54 & 0.000 \\
\hline Midline & 0.83 & 32.86 & 6.49 & 0.011 \\
\hline Buccal occlusion & 0.47 & 194.33 & 76.56 & 0.000 \\
\hline Upper anterior crowding & 0.25 & 102.56 & 30.10 & 0.000 \\
\hline $\mathrm{R}$ square & 0.57 & & & \\
\hline
\end{tabular}

Equation: Anticipated duration of treatment $=3.2+0.96$ (overjet) +0.75 (overbite) +0.83 (midline) +0.47 (buccal occlusion) +0.25 (upper anterior crowding).

Table IV. The weightings for the components of PAR for malocclusion severity and treatment difficulty

\begin{tabular}{l|c|c|c}
\hline Component of malocclusion & Severity weighting & Difficulty weighting & Combined weighting \\
\hline Overjet & 5 & 4 & 4.5 \\
Overbite & 3 & 3 & 3 \\
Midline discrepancy & 3 & 4 & 3.5 \\
Buccal occlusion & 2 & 2 & 2 \\
Upper anterior alignment & 1 & 1 & 1 \\
\hline
\end{tabular}

ceived malocclusion severity to $r=0.8$ and for treatment difficulty to $r=0.65$.

\section{The comparison of the scores for differing levels of severity and difficulty}

Table $\mathrm{V}$ contains the means and standard deviations of the weighted PAR scores for the perceived levels of malocclusion severity, and perceived treatment difficulty. Analysis of variance revealed that there were significant differences between the PAR scores associated with the three levels of malocclusion severity and treatment difficulty $(p<0.001)$. The Tukey multiple comparison test revealed that the differences in scores between the groups were all highly significant $(p<0.001)$.

\section{DISCUSSION}

One of the major findings of this investigation was that there was a close association between orthodontists' perception of severity of dental malocclusion and perceived difficulty of treatment, and they may in fact not be entirely separate variables. As a result, it is likely that any measure of malocclusion severity will be essentially evaluating the same features as a measure of treatment difficulty. This investigation has resulted in the development of one measure that reflects both malocclusion severity and treatment difficulty.

However, the difficulty of treatment if estimated before treatment cannot make a definite estimate of individual variability in treatment response, that may be a result of biologic and/or psychosocial
Table V. The weighted PAR scores for the levels of malocclusion severity and treatment difficulty. (Means with standard deviations in parentheses)

\begin{tabular}{l|c}
\hline & Weighted PAR scores \\
\hline Malocclusion severity & $17.17(2.34)$ \\
Mild & $39.79(1.29)$ \\
Moderate & $53.56(1.38)$ \\
Severe & \\
& \\
Treatment difficulty & \\
$\quad$ Very easy & $25.32(3.03)$ \\
Moderately difficult & $43.59(1.25)$ \\
Very difficult & $55.26(1.57)$ \\
\hline
\end{tabular}

attributes of patients. As a result, only estimates of "anticipated" difficulty or duration can be made a priori. Therefore the next stage in the development of this measure will involve its application to a sample of treated cases to determine whether factors can be identified that can improve the a priori prediction of treatment difficulty.

It was also evident that when the panel made their assessments, they placed an emphasis on certain morphologic features of malocclusion, namely, overjet, overbite, midline discrepancy, upper anterior alignment, and buccal segment relationships. In fact, lower incisor and buccal segment crowding did not have a predictive effect. Further examination of the relative weightings reveals an order of ranking of 
malocclusion components with which few orthodontists would disagree, both in terms of malocclusion severity and treatment difficulty. Nevertheless, the absence of weighting for buccal segment and lower anterior crowding is difficult to explain. One reasonable explanation may be that because crowding in the buccal and lower anterior segments is associated with other features of malocclusion; these and not crowding were the primary concerns of the panel. On the difficulty of treatment, opinions may have been influenced by the comparative ease with which dental crowding is corrected by extraction of teeth or arch expansion, in contrast to the more difficult correction of overjet or midline discrepancy.

\section{Limitations of occlusal indices as measures of outcome for orthodontics}

Although the use of occlusal indices is a quick, valid, and accurate method of assessing the dentoocclusal aspects of orthodontic treatment results, there are admittedly some limitations. Importantly, changes in facial profile, or cephalometric parameters that reflect the skeletal component of malocclusion are not considered in the quantitative evaluation. Unfortunately, measurement of these variables by valid and reliable methods has not been achieved for the following reasons. First, individal biologic variation requires discrimination between changes attributable to orthodontic intervention and those due to the growth and development of the facial complex. Second, the ideal cephalomeric analysis or cephalometric goals of orthodontic treatment are controversial with no consensus throughout the profession. Finally, no universally accepted methods currently exist to assess change in facial profile as an outcome measure. For the time being, the optimal feasible method for assessing the attainment of desired occlusal outcomes is by the use of occlusal indices. This procedure enables an accurate evaluation of many of the effects of treatment.

\section{Limitations of this investigation}

These results were derived from a panel of orthodontists practicing in western Pennsylvania. It is possible that the perception of malocclusion and treatment difficulty may vary between different geographic locations in the United States and further validation exercises may be indicated. In addition, orthodontists may use information obtained from diagnostic records other than study casts in deriving perceptions of malocclusion severity and treatment difficulty. Consequently, the results may vary when additional clinical information is made available. Nevertheless, at least $65 \%$ of the variation in data was explained by the weighted PAR index, which suggests that the addition of further records may not lead to a greater information yield. Consequently, until further investigations that evaluate the validity of the PAR Index either refute or support these findings, an important perception relevant to clinical practice has emerged.

\section{CONCLUSION}

The results of this study made it possible to derive a set of weightings for the PAR index and to calculate scores that would represent groupings of malocclusions severity and treatment difficulty, according to the perceptions of a panel of orthodontists. As a result, the PAR index may be considered to represent a good approximation of malocclusion severity and treatment difficulty, and may be used as an outcome measure for the assessment of dentoocclusal change, in studies investigating the effectiveness of orthodontic treatment that are based in the United States.

We are indebted to the group of orthodontic raters who kindly gave their time and expertise to score the models on two occasions. We recognize that this involved sacrificing time from their practices for which we are most grateful.

\section{REFERENCES}

1. Shaw WC, Richmond S, O'Brien KD, Brook PH, Stephens $\mathrm{CD}$. Indices of orthodontic treatment need and standards. $\mathrm{Br}$ Dent J 1991;170:107-12.

2. Summers $C J$. The occlusal index: a system for identifying and scoring occlusal disorders. AM J ORTHOD 1971;59:552-66.

3. Richmond S, Shaw WC, Stephens CD, et al. The PAR index, reliability and validity. Eur J Orthod 1992;14:125-39.

4. Pickering EA, Vig PS. The occlusal index used to assess orthodontic treatment. Br J Orthod 1975;2:47-51.

5. Elderton RJ, Clark JD. Orthodontic treatment in the general dental service assessed by the occlusal index. $\mathrm{Br} \mathrm{J}$ Orthod 1983;10:178-86.

6. O'Brien KD, Shaw WC, Roberts CT. An evaluation of the effectiveness of the hospital orthodontic service. Br J Orthod 1993;20:25-35.

7. Rowe KGT. The concordance of pre-treatment malocclusion assessment among orthodontic specialty practitioners. [Master's thesis]. Ann Arbor: University of Michigan, 1989.

\section{Reprint requests to:}

Dr. K. O'Brien

Department of Dental Medicine and Surgery

University Dental School of Manchester

Higher Cambridge St.

Manchester M15 6FH

England 